\title{
ASSESSMENT OF SELECTED FOOD INTAKE FREQUENCY IN PATIENTS WITH TYPE 1 DIABETES TREATED WITH PERSONAL INSULIN PUMPS
}

\author{
Sabina Krzyżowska ${ }^{1}$, Bartłomiej Matejko ${ }^{1,2}$, Beata Kieć-Wilk ${ }^{1,2}$, Magdalena Wilk ${ }^{1,2}$ \\ Maciej Małecki ${ }^{1,2}$, Tomasz Klupa ${ }^{1,2}$ \\ ${ }^{1}$ Department of Metabolic Diseases, University Hospital, Krakow, Poland \\ ${ }^{2}$ Department of Metabolic Diseases, Jagiellonian University Medical College, Krakow, Poland
}

\begin{abstract}
Background. It has been established that in Type 1 Diabetes Mellitus (T1DM), regardless of the insulin therapy model used, diet and proper eating habits are still important in the treatment of the disease. The dietary intervention in these patients is aimed at maintaining proper body weight, obtaining target fasting and post meal blood glucose levels, optimizing lipid profiles.

Objective. The aim of the study was to assess dietary habits in a homogeneous group of adults with T1DM treated with personal insulin pumps.

Material and methods. The study included 141 adult patients (57\% women) with type 1 diabetes treated with personal insulin pumps. The surveyed population was characterized by an average age of $25.8 \pm 6.2$ years, an average duration of diabetes $13.9 \pm 6.9$ years, and treatment with a personal pump for $8.2 \pm 4.1$ years and mean BMI $23.0 \pm 2.8 \mathrm{~g} / \mathrm{m}^{2}$. All were dwellers of south-eastern Poland. The validated KomPAN questionnaire was used to assess the frequency of consumption of individual food products.

Results. The mean percentage of HbAlc in the study group was 7.3\% [56 $\mathrm{mmol} / \mathrm{mol}]$. The mean total cholesterol level was $4.4 \mathrm{mmol} / \mathrm{l}$, HDL $-1.7 \mathrm{mmol} / \mathrm{l}, \mathrm{LDL}-2.3 \mathrm{mmol} / 1$ and triglycerides $-0.8 \mathrm{mmol} / \mathrm{l}$. In the multivariate regression model, no correlation was found between dietary quality parameters and metabolic compensation measured with $\mathrm{HbAlc}$ or lipidogram and the place of residence (village, small town, big city). However, there were differences in the quality of the diet depending on the sex. Women were characterized by higher index of a healthy diet (pHDI-10) (26.3 vs $21.4, \mathrm{p}=0.005)$ and lower index of unhealthy diet (nHDI-14) $(13.3$ vs $18.6, \mathrm{p}<0.001)$ than men.

Conclusions. The results of this study clearly suggest, that despite good metabolic control, patients require more education on the choice of healthy product groups.
\end{abstract}

Key words: diabetes type 1, diet, personal insulin pump

\section{STRESZCZENIE}

Wprowadzenie. Uważa się, że w cukrzycy typu 1 (T1DM), niezależnie od zastosowanego modelu insulinoterapii, dieta i prawidłowe nawyki żywieniowe są kluczowe w leczeniu cukrzycy. Stosowanie odpowiedniej diety ma na celu utrzymanie prawidłowej masy ciała, uzyskanie prawidłowego poziomu glukozy we krwi po posiłku, optymalnego profil lipidowy i prawidłowe wartości ciśnienia krwi.

Cel. Celem badania była ocena diety i kontrola metaboliczna w homogennej grupie osób dorosłych z T1DM leczonych osobistymi pompami insulinowymi.

Materiał i metody: Badaniem objęto 141 dorosłych pacjentów (57\% kobiet) z cukrzycą typu 1 leczonych osobistymi pompami insulinowymi. Badana populacja charakteryzowała się średnim wiekiem 25,8 $\pm 6,2$ lat, średnim czasem trwania cukrzycy 13,9 $\pm 6,9$ lat oraz średnim czasem leczenia osobistą pompą przez 8,2 $\pm 4,1$ lat i średnim BMI 23,0 $\pm 2,8 \mathrm{~g} /$ $\mathrm{m} 2$. Wszyscy byli mieszkańcami południowo-wschodniej Polski. Walidowany kwestionariusz KomPAN wykorzystano do oceny częstotliwości spożycia poszczególnych produktów spożywczych.

Wyniki. Średni odsetek HbA1c w grupie badanej wynosił 7,3\% [56 mmol/mol]. Średni poziom całkowitego cholesterolu wynosił 4,4 mmol / 1, HDL - 1,7 mmol / 1, LDL - 2,3 mmol / 1 i triglicerydów - 0,8 mmol / 1 . W modelu regresji wielorakiej nie stwierdzono korelacji między wskaźnikami diety i wyrównaniem metabolicznym mierzonym za pomocą HbAlc lub lipidogramu oraz miejscem zamieszkania (wieś, małe miasteczko, duże miasto). Jednak różnice w jakości diety zależały

Corresponding author: Tomasz Klupa, Department of Metabolic Diseases, Jagiellonian University Medical College, 15 Kopernika Street, 31-501 Krakow, Poland, Tel.: +48 4248 300, e-mail: tomasz.klupa@uj.edu.pl

(C) Copyright by the National Institute of Public Health - National Institute of Hygiene 
od płci. Kobiety charakteryzowały się wyższym wskaźnikiem zdrowej diety (pHDI-10) $(26,3$ vs 21,4, p =0,005) i niższym wskaźnikiem niezdrowej diety (nHDI-14) $(13,3$ vs $18,6, \mathrm{p}<0,001)$ niż mężczyźni.

Wnioski: Wyniki tego badania wyraźnie wskazują, że pomimo dobrej kontroli metabolicznej pacjenci wymagają większej edukacji w zakresie wyboru zdrowych grup produktów.

Slowa kluczowe: cukrzyca typu 1, dieta, osobista pompa insulinowa

\section{INTRODUCTION}

Type 1 diabetes mellitus (T1DM) is a disorder resulting from an autoimmune destruction of the pancreatic beta cells and characterized by a decline in and later an absence of insulin production by the pancreas which leads to absolute insulin deficiency. Treatment of T1DM involves replacement of insulin in an effort to achieve blood glucose levels that are as close as possible to the normal range with the main goal to prevent acute and late complications of diabetes. Medical nutrition therapy is also an important component of modern diabetes treatment $[1,2]$.

Definitely the key component of dietary management of T1DM is monitoring carbohydrate intake and appropriately adjusting insulin doses [1]. Apart from that nutrition guidelines in type 1 diabetes is not essentially different in terms of the principles of rational nutrition from the general population of healthy people $[1,2]$. However with the lifestyle flexibility offered by modern developments in diabetes management, like personal insulin pumps, individuals with T1DM are often tempted not to follow general recommendations of healthy diet. This may be of importance, especially in the context of development of long term macrovascular complications of the disease $[7,11]$.

Our cross-sectional study aimed to assess diet composition, implementation of dietary recommendations and their relation to metabolic control in a homogeneous group of adults with T1DM treated with personal insulin pumps.

\section{METHODS}

The study included 141 adult patients $(57 \%$ of the group were women) with type 1 diabetes treated with personal insulin pumps, under the constant follow-up at the Department of Metabolic Diseases, University Hospital in Krakow. The surveyed population was characterized by an average age of $25.8 \pm 6.2$ years, an average duration of diabetes $13.9 \pm 6.9$ years, and treatment with a personal pump for $8,2 \pm 4.1$ years and mean BMI $23.0 \pm 2.8 \mathrm{~g} / \mathrm{m}^{2}$. The study group included people with diabetes diagnosed more than 10 years ago (67\%), 5-10 years ago (21\%) and less than 5 years $(12 \%)$. All were dwellers of south-eastern Poland: $35 \%$ lived in the villages, $25 \%$ in towns below 100,000 residents and $40 \%$ in a city of over 100 inhabitants (Krakow). The study had been approved by the Bioethical Commission at the Jagiellonian University No. 122.6120.50.2017, all the participants signed written consent.

The exclusion criteria included: pregnancy, treatment with multiple insulin injections, i.e. pens, presence of advanced late complications of diabetes, i.e. retinopathy, nephropathy and neuropathy.

Following data were analyzed during the study:

1) questionnaire data,

2) data from the insulin pump and glucose meter downloads (based on reports generated by the dedicated software Accu Chek 360 - Roche and CareLink Proffesional - Medtronic). The data from one moth prior to the visit date were analysed

3) the last available $\mathrm{HbA} 1 \mathrm{c}$ and lipid profiles in the patient's medical base.

In addition, three indicators were calculated based on lipid profile parameters: TC/HDL ratio - as an indicator of coronary risk factor [3], LDL/HDL ratio [9] and TG/HDL ratio as a predictor of insulin resistance [15].

Variables obtained from personal insulin pump and glucometer reports included: mean glycemia, standard glycemic deviation, daily insulin dose, percentage of basal insulin and the number of hypoglycaemic episodes in the analyzed period divided by the number of days analyzed (28 days prior to clinic visit were used for analysis). For the purpose of the study we used 40 questions derived from the KomPAN questionnaire, which is an improved and extended version of the QEB (Beliefs and Eating Habits Questionnaire) questionnaire and was created by Behavioral Conditions of Nutrition Team, Committee of Human Nutrition Science, Polish Academy of Science. For the purpose of this study the questionnaire had been extended with 13 additional, original questions [8]. All the data were analysed with respect to gender since pre study initial evaluation of the study group pointed to possible gender-dependent differences.

In order to comprehensively evaluate the quality of the diet, two diet index were calculated:

- Index of a healthy diet (pHDI-10, Prohealthy-DietIndex-10) calculated by summing the frequency of consumption (multiplicity / day): fruit, vegetables, wholemeal bread, milk (including flavored milk), 
milky fermented beverages (like yoghurts, kefirs)), curd cheese (including homogenized cheese), fish products and dishes, legume dishes, thick milled buckwheat cereal products, oatmeal, whole-grain pasta and other coarse groats and dishes from the so-called white meat, e.g. chicken, turkey, rabbit;

- Index of unhealthy diet (nHDI-14, NonHealthy-Diet-Index-14) calculated by summing the frequency of consumption (times / day): confectionery,, fried dishes, alcoholic drinks, sweetened carbonated beverages, meat, powder soups and ready-to-eat soups, fast food and energy drinks, light breads, "white" cereal products white rice, plain pasta or small groats, semolina, couscous; butter, lard, yellow cheese, red meat dishes, veal, mutton, lamb, beef, pork or venison.

\section{Statistical analysis}

The SPSS package was used for statistical analysis. To check if variables have a normal distribution, the Shapiro-Wilk test was used. The t-Student test and its non-parametric counterpart, the Mann-Whitney test, were used to analyze quantitative variables in two subgroups, and the chi ${ }^{2}$ test was used to analyze qualitative data. To check whether the obtained results are not disturbed by the influence of age, gender and BMI index, a multiple regression analysis was used. Statistically significant results were those of $p<0.05$.

\section{RESULTS}

Table 1 presents the characteristics of the studied group (for the whole population and with respect to gender). Table 2 presents the frequency of consumption of selected food groups with respect to gender.

Table 1. Characteristics of the study population with respect to gender

\begin{tabular}{|c|c|c|c|c|c|c|c|c|}
\hline \multirow{2}{*}{ Variables } & \multirow{2}{*}{$\mathrm{N}$} & \multicolumn{2}{|c|}{ Overall } & \multicolumn{2}{|c|}{ Women } & \multicolumn{2}{|c|}{ Men } & \multirow{2}{*}{$\mathrm{p}$} \\
\hline & & Mean & SD & Mean & SD & Mean & SD & \\
\hline Age [year] & 141 & 25.79 & 6.16 & 26.32 & 6.44 & 25.07 & 5.73 & 0.260 \\
\hline BMI $\left[\mathrm{kg} / \mathrm{m}^{2}\right]$ & 141 & 23.01 & 2.81 & 22.68 & 2.73 & 23.47 & 2.87 & 0.068 \\
\hline Pump treatment duration [year] & 138 & 8.23 & 4.14 & 8.15 & 4.32 & 8.35 & 3.91 & 0.769 \\
\hline T1DM duration [years] & 138 & 13.88 & 6.90 & 15.13 & 17.22 & 14.59 & 7.37 & 0.488 \\
\hline Average glycemia from glucose meter [mg/dl] & 139 & 157.88 & 32.61 & 154.23 & 33.03 & 162.98 & 31.57 & 0.060 \\
\hline Standard devation of glycemia from glucose meter [mg/dl] & 137 & 73.35 & 20.09 & 70.83 & 19.73 & 76.99 & 20.23 & 0.078 \\
\hline Number of glucometer measurements per day [n] & 137 & 5.42 & 3.25 & 5.52 & 3.52 & 5.29 & 2.87 & 0.848 \\
\hline DID [IU] & 136 & 46.57 & 14.15 & 42.91 & 11.42 & 51.49 & 15.97 & 0.001 \\
\hline$\% \mathrm{BI}[\%]$ & 136 & 44.60 & 10.47 & 46.40 & 11.24 & 42.17 & 8.87 & 0.019 \\
\hline Number of hypoglycemia per day [n] & 140 & 0.50 & 0.47 & 0.52 & 0.49 & 0.46 & 0.43 & 0.487 \\
\hline $\mathrm{HbA}_{1 \mathrm{c}}[\mathrm{mmol} / \mathrm{l}]$ & 141 & 7.36 & 1.16 & 7.50 & 1.22 & 7.17 & 1.04 & 0.151 \\
\hline $\mathrm{TC}[\mathrm{mmol} / \mathrm{l}]$ & 140 & 4.36 & 0.71 & 4.52 & 0.75 & 4.14 & 0.59 & 0.001 \\
\hline HDL [mmol/l] & 141 & 1.69 & $\mathbf{0 . 3 8}$ & 1.80 & 0.38 & 1.54 & $\mathbf{0 . 3 3}$ & $<0.001$ \\
\hline LDL $[\mathrm{mmol} / \mathrm{l}]$ & 140 & 2.28 & 0.59 & 2.30 & 0.62 & 2.25 & 0.55 & 0.649 \\
\hline TG $[\mathrm{mmol} / \mathrm{l}]$ & 140 & 0.83 & 0.38 & 0.85 & 0.41 & 0.81 & 0.35 & 0.930 \\
\hline TC/HDL [mmol/l] & 141 & 2.66 & 0.69 & 2.55 & 0.67 & 2.79 & 0.69 & 0.059 \\
\hline LDL/HDL [mmol/1] & 141 & 1.41 & 0.55 & 1.31 & 0.51 & 1.54 & 0.57 & 0.012 \\
\hline TG/HDL [mmol/l] & 141 & 0.53 & 0.32 & 0.46 & 0.30 & 0.57 & 0.34 & 0.075 \\
\hline pHDI-10 [pkt] & 141 & 24.22 & 10.28 & 26.31 & 10.83 & 21.39 & 8.80 & 0.005 \\
\hline nHDI-14 [pkt] & 141 & 15.56 & 7.61 & 13.32 & 6.77 & 18.59 & 7.68 & $<0.001$ \\
\hline
\end{tabular}

BMI- body mass index; DID- Daily Insulin Dose; \%BI- percentage of basal insulin; IU-insulin units; $\mathrm{HBA}_{1 \mathrm{c}}-$ hemoglobin $\mathrm{A}_{1 \mathrm{c}}$; TC- total cholesterol; LDL- low-density lipoprotein; HDL- high-density lipoprotein; TG- triglycerides.

In studied population, 3\% of people consumed only 2 meals a day, $25 \%$ consumed 3 meals, $52 \%$ - 4 meals, and only $21 \%$ consumed 5 or more meals a day. Only $18 \%$ of respondents ate all meals at regular times, $56 \%$ regularly consumed some meals, other people ate meals irregularly. $74.5 \%$ of diabetics declared that they would eat between the main meals. Most often they chose: fruits (59\%) and nuts and other seeds $40 \%$ ). About $1 / 4$ of people reported that they eat sweet snacks between meals, e.g. candies, cookies, cakes, chocolate bars, muesli bars, wafers or unsweetened drinks and milk desserts, e.g. yoghurts, cottage cheese, milk or vegetables.
In the study results analysis first we tried to find correlation between diet quality parameters and $\mathrm{HbA} 1 \mathrm{c}$ but we failed to find one in the multivariate regression model. There was also no relationship between the quality of the diet and the place of residence (village, small town, big city).

Also linear and multivariate regression were performed to check for correlations between diet and lipid profile components. The pHDI-10 was associated with HDL cholesterol $(b=0.006, p=0.046)$ and the TG / HDL ratio $(b=-0.005 ; \mathrm{p}=0.025)$, while the nHDI14 ratio was associated only with total cholesterol 
$(b=-0.02, p=0.007)$. Taking that under consideration, the 0.024 model, but only nHDI-14 affects: $b=-0.019$, the relationship between the two indicators taken together $p=0.007$ ) and for HDL ( $p$ for the model 0.03, but the and the components of the lipid profile were analyzed. indicators were not shown to be statistically significant.

The dependency was shown for total cholesterol ( $\mathrm{p}$ for

Table 2. Frequency of consumption of selected groups of food products and meals outside the home - expressed as a percentage $(\%)$

\begin{tabular}{|c|c|c|c|c|c|}
\hline Food products & $\begin{array}{c}\text { Frequency of consumption } \\
\text { in } 4 \text { categories }\end{array}$ & Overall & Women & Men & $\mathrm{p}$ \\
\hline Wholemeal bread and rolls & $\begin{array}{l}\text { Seldom/never } \\
\text { Once per week } \\
\text { Several time per week } \\
\text { Every day }\end{array}$ & $\begin{array}{l}36.2 \\
12.1 \\
30.5 \\
21.3\end{array}$ & $\begin{array}{l}38.3 \\
11.1 \\
29.6 \\
21.0\end{array}$ & $\begin{array}{l}33.3 \\
13.3 \\
31.7 \\
21.7\end{array}$ & NS \\
\hline White bread and rolls & $\begin{array}{l}\text { Seldom/never } \\
\text { Once per week } \\
\text { Several time per week } \\
\text { Every day }\end{array}$ & $\begin{array}{c}24.1 \\
8.5 \\
32.6 \\
34.8 \\
\end{array}$ & $\begin{array}{c}33.3 \\
8.6 \\
29.6 \\
28.4 \\
\end{array}$ & $\begin{array}{c}11.7 \\
8.3 \\
36.7 \\
43.3 \\
\end{array}$ & 0.024 \\
\hline $\begin{array}{l}\text { Cereal products such as white rice, plain } \\
\text { pasta or fine groats }\end{array}$ & $\begin{array}{l}\text { Seldom/never } \\
\text { Once per week } \\
\text { Several time per week } \\
\text { Every day }\end{array}$ & $\begin{array}{c}27.0 \\
28.4 \\
39.6 \\
5.0\end{array}$ & $\begin{array}{c}30.9 \\
28.4 \\
37.0 \\
3.7\end{array}$ & $\begin{array}{c}21.7 \\
28.3 \\
43.3 \\
6.7 \\
\end{array}$ & NS \\
\hline $\begin{array}{l}\text { Grain products such as buckwheat, } \\
\text { oatmeal, whole-grain pasta or other whole } \\
\text { grain groats }\end{array}$ & $\begin{array}{l}\text { Seldom/never } \\
\text { Once per week } \\
\text { Several time per week } \\
\text { Every day }\end{array}$ & $\begin{array}{l}46.1 \\
19.1 \\
31.9 \\
26.2\end{array}$ & $\begin{array}{c}48.1 \\
16.0 \\
32.7 \\
3.7\end{array}$ & $\begin{array}{c}43.3 \\
23.3 \\
31.7 \\
1.7\end{array}$ & NS \\
\hline Milk (including flavored milk) & $\begin{array}{l}\text { Seldom/never } \\
\text { Once per week } \\
\text { Several time per week } \\
\text { Every day } \\
\end{array}$ & $\begin{array}{l}29.1 \\
12.8 \\
31.9 \\
26.2 \\
\end{array}$ & $\begin{array}{c}27.2 \\
7.4 \\
33.3 \\
32.1 \\
\end{array}$ & $\begin{array}{l}31.7 \\
20.0 \\
30.0 \\
18.3 \\
\end{array}$ & 0.068 \\
\hline Fermented milk drinks, e.g. yogurts, kefirs & $\begin{array}{l}\text { Seldom/never } \\
\text { Once per week } \\
\text { Several time per week } \\
\text { Every day } \\
\end{array}$ & $\begin{array}{l}22.7 \\
19.1 \\
39.0 \\
19.1 \\
\end{array}$ & $\begin{array}{l}17.3 \\
22.2 \\
38.3 \\
22.2 \\
\end{array}$ & $\begin{array}{l}30.0 \\
15.0 \\
40.0 \\
15.0 \\
\end{array}$ & NS \\
\hline Cottage cheese (including cream cheese) & $\begin{array}{l}\text { Seldom/never } \\
\text { Once per week } \\
\text { Several time per week } \\
\text { Every day } \\
\end{array}$ & $\begin{array}{c}33.3 \\
17.0 \\
40.4 \\
9.2 \\
\end{array}$ & $\begin{array}{l}25.9 \\
19.8 \\
43.2 \\
11.1\end{array}$ & $\begin{array}{c}43.3 \\
13.3 \\
36.7 \\
6.7 \\
\end{array}$ & NS \\
\hline $\begin{array}{l}\text { Yellow cheese (including proccesed } \\
\text { cheese) }\end{array}$ & $\begin{array}{l}\text { Seldom/never } \\
\text { Once per week } \\
\text { Several time per week } \\
\text { Every day } \\
\end{array}$ & $\begin{array}{l}14.2 \\
19.9 \\
46.1 \\
19.9 \\
\end{array}$ & $\begin{array}{l}19.8 \\
22.2 \\
42.0 \\
16.0\end{array}$ & $\begin{array}{c}6.7 \\
16.7 \\
51.7 \\
25.0 \\
\end{array}$ & 0.079 \\
\hline $\begin{array}{l}\text { Butter as an addition to breads or dishes, } \\
\text { for frying, baking, etc.. }\end{array}$ & $\begin{array}{l}\text { Seldom/never } \\
\text { Once per week } \\
\text { Several time per week } \\
\text { Every day } \\
\end{array}$ & $\begin{array}{c}20.6 \\
6.4 \\
30.5 \\
42.6\end{array}$ & $\begin{array}{c}18.5 \\
7.4 \\
33.3 \\
40.7 \\
\end{array}$ & $\begin{array}{c}23.3 \\
5.0 \\
26.7 \\
45.0\end{array}$ & NS \\
\hline $\begin{array}{l}\text { Oil or margarines or butter mixes with } \\
\text { margarine, in addition to breads or dishes, } \\
\text { for frying, baking, etc. }\end{array}$ & $\begin{array}{l}\text { Seldom/never } \\
\text { Once per week } \\
\text { Several time per week } \\
\text { Every day } \\
\end{array}$ & $\begin{array}{l}50.4 \\
13.5 \\
22.7 \\
13.5 \\
\end{array}$ & $\begin{array}{c}55.6 \\
14.8 \\
19.8 \\
9.9 \\
\end{array}$ & $\begin{array}{l}43.3 \\
11.7 \\
26.7 \\
18.3 \\
\end{array}$ & NS \\
\hline Ham, sausages & $\begin{array}{l}\text { Seldom/never } \\
\text { Once per week } \\
\text { Several time per week } \\
\text { Every day } \\
\end{array}$ & $\begin{array}{l}12.1 \\
15.0 \\
45.0 \\
27.9\end{array}$ & $\begin{array}{l}11.3 \\
16.3 \\
50.0 \\
22.5 \\
\end{array}$ & $\begin{array}{l}13.3 \\
13.3 \\
38.3 \\
35.0 \\
\end{array}$ & NS \\
\hline $\begin{array}{l}\text { Red meat, e.g. pork, beef, veal, mutton, } \\
\text { lamb and venison }\end{array}$ & $\begin{array}{l}\text { Seldom/never } \\
\text { Once per week } \\
\text { Several time per week } \\
\text { Every day }\end{array}$ & $\begin{array}{c}44.3 \\
27.9 \\
25.7 \\
2.1 \\
\end{array}$ & $\begin{array}{c}53.8 \\
25.0 \\
20.0 \\
1.3 \\
\end{array}$ & $\begin{array}{c}31.7 \\
31.7 \\
33.3 \\
3.3 \\
\end{array}$ & 0.061 \\
\hline White meat, e.g. chicken, turkey, rabbit & $\begin{array}{l}\text { Seldom/never } \\
\text { Once per week } \\
\text { Several time per week } \\
\text { Every day }\end{array}$ & $\begin{array}{c}12.1 \\
15.7 \\
65.0 \\
7.1 \\
\end{array}$ & $\begin{array}{c}11.3 \\
16.3 \\
67.5 \\
5.0 \\
\end{array}$ & $\begin{array}{l}13.3 \\
15.0 \\
61.7 \\
10.0 \\
\end{array}$ & NS \\
\hline Fishes & $\begin{array}{l}\text { Seldom/never } \\
\text { Once per week } \\
\text { Several time per week } \\
\text { Every day }\end{array}$ & $\begin{array}{c}43.3 \\
41.8 \\
14.2 \\
0.7 \\
\end{array}$ & $\begin{array}{c}39.5 \\
43.2 \\
16.0 \\
1.2 \\
\end{array}$ & $\begin{array}{c}48.3 \\
40.0 \\
11.7 \\
0.0 \\
\end{array}$ & NS \\
\hline Eggs & $\begin{array}{l}\text { Seldom/never } \\
\text { Once per week } \\
\text { Several time per week } \\
\text { Every day }\end{array}$ & $\begin{array}{c}26.2 \\
31.9 \\
38.3 \\
3.5 \\
\end{array}$ & $\begin{array}{c}23.5 \\
35.8 \\
38.3 \\
2.5 \\
\end{array}$ & $\begin{array}{c}30.0 \\
26.7 \\
38.3 \\
5.0 \\
\end{array}$ & NS \\
\hline Fruits & $\begin{array}{l}\text { Seldom/never } \\
\text { Once per week } \\
\text { Several time per week } \\
\text { Every day }\end{array}$ & $\begin{array}{c}4.3 \\
7.8 \\
34.8 \\
53.2 \\
\end{array}$ & $\begin{array}{c}2.5 \\
8.6 \\
30.9 \\
58.0 \\
\end{array}$ & $\begin{array}{c}6.7 \\
6.7 \\
40.0 \\
46.7 \\
\end{array}$ & NS \\
\hline
\end{tabular}




\begin{tabular}{|c|c|c|c|c|c|}
\hline Food products & $\begin{array}{l}\text { Frequency of consumption } \\
\text { in } 4 \text { categories }\end{array}$ & Overall & Women & Men & $\mathrm{p}$ \\
\hline Vegetables & $\begin{array}{l}\text { Seldom/never } \\
\text { Once per week } \\
\text { Several time per week } \\
\text { Every day }\end{array}$ & $\begin{array}{c}1.4 \\
2.1 \\
39.0 \\
57.4 \\
\end{array}$ & $\begin{array}{c}0.0 \\
1.2 \\
30.9 \\
67.9 \\
\end{array}$ & $\begin{array}{c}3.3 \\
3.3 \\
50.0 \\
43.3 \\
\end{array}$ & 0.016 \\
\hline Legumes & $\begin{array}{l}\text { Seldom/never } \\
\text { Once per week } \\
\text { Several time per week } \\
\text { Every day } \\
\end{array}$ & $\begin{array}{c}61.0 \\
19.1 \\
18.4 \\
1.4 \\
\end{array}$ & $\begin{array}{c}55.6 \\
21.0 \\
21.0 \\
2.5 \\
\end{array}$ & $\begin{array}{c}68.3 \\
16.7 \\
15.0 \\
0.0 \\
\end{array}$ & NS \\
\hline Potatoes & $\begin{array}{l}\text { Seldom/never } \\
\text { Once per week } \\
\text { Several time per week } \\
\text { Every day }\end{array}$ & $\begin{array}{l}17.7 \\
19.1 \\
51.8 \\
11.3\end{array}$ & $\begin{array}{c}22.2 \\
23.5 \\
45.7 \\
8.6\end{array}$ & $\begin{array}{l}11.7 \\
13.3 \\
60.0 \\
15.0\end{array}$ & 0.086 \\
\hline $\begin{array}{l}\text { Fast food, such as fries, hamburgers, } \\
\text { pizzas, hot dogs, casseroles }\end{array}$ & $\begin{array}{l}\text { Seldom/never } \\
\text { Once per week } \\
\text { Several time per week } \\
\text { Every day }\end{array}$ & $\begin{array}{c}79.4 \\
14.9 \\
5.7 \\
0.0\end{array}$ & $\begin{array}{c}86.4 \\
12.3 \\
1.2 \\
0.0\end{array}$ & $\begin{array}{c}70.0 \\
18.3 \\
11.7 \\
0.0\end{array}$ & 0.013 \\
\hline $\begin{array}{l}\text { Sweets, e.g. sweets, cakes, chocolate bars, } \\
\text { muesli bars, other confectionery }\end{array}$ & $\begin{array}{l}\text { Seldom/never } \\
\text { Once per week } \\
\text { Several time per week } \\
\text { Every day } \\
\end{array}$ & $\begin{array}{l}27.0 \\
19.9 \\
40.4 \\
12.8 \\
\end{array}$ & $\begin{array}{c}32.1 \\
24.7 \\
35.8 \\
7.4 \\
\end{array}$ & $\begin{array}{l}20.0 \\
13.3 \\
46.7 \\
20.0 \\
\end{array}$ & 0.024 \\
\hline Powdered soups or ready-made soups & $\begin{array}{l}\text { Seldom/never } \\
\text { Once per week } \\
\text { Several time per week } \\
\text { Every day } \\
\end{array}$ & $\begin{array}{c}93.6 \\
4.3 \\
2.1 \\
0.0 \\
\end{array}$ & $\begin{array}{l}92.6 \\
4.9 \\
2.5 \\
0.0\end{array}$ & $\begin{array}{l}95.0 \\
3.3 \\
1.7 \\
0.0 \\
\end{array}$ & NS \\
\hline Canned meat, fish, vegetables and meat & $\begin{array}{l}\text { Seldom/never } \\
\text { Once per week } \\
\text { Several time per week } \\
\text { Every day }\end{array}$ & $\begin{array}{l}89.4 \\
8.5 \\
2.1 \\
0.0 \\
\end{array}$ & $\begin{array}{l}93.8 \\
6.2 \\
0.0 \\
0.0 \\
\end{array}$ & $\begin{array}{c}83.3 \\
11.7 \\
5.0 \\
0.0 \\
\end{array}$ & 0.058 \\
\hline $\begin{array}{l}\text { Canned vegetables or fruits, marinades, } \\
\text { silage }\end{array}$ & $\begin{array}{l}\text { Seldom/never } \\
\text { Once per week } \\
\text { Several time per week } \\
\text { Every day }\end{array}$ & $\begin{array}{c}75.9 \\
17.7 \\
6.4 \\
0.0 \\
\end{array}$ & $\begin{array}{c}67.9 \\
24.7 \\
7.4 \\
0.0 \\
\end{array}$ & $\begin{array}{l}86.7 \\
8.3 \\
5.0 \\
0.0 \\
\end{array}$ & 0.029 \\
\hline Fried dishes & $\begin{array}{l}\text { Seldom/never } \\
\text { Once per week } \\
\text { Several time per week } \\
\text { Every day } \\
\end{array}$ & $\begin{array}{c}25.5 \\
39.0 \\
33.3 \\
2.1 \\
\end{array}$ & $\begin{array}{c}29.6 \\
44.4 \\
24.7 \\
1.2 \\
\end{array}$ & $\begin{array}{c}20.0 \\
31.7 \\
45.0 \\
3.3 \\
\end{array}$ & 0.053 \\
\hline Water, e.g. mineral, table water & $\begin{array}{l}\text { Seldom/never } \\
\text { Once per week } \\
\text { Several time per week } \\
\text { Every day }\end{array}$ & $\begin{array}{c}0.7 \\
0.0 \\
6.4 \\
92.9 \\
\end{array}$ & $\begin{array}{c}1.2 \\
0.0 \\
2.5 \\
96.3 \\
\end{array}$ & $\begin{array}{c}0.0 \\
0.0 \\
11.7 \\
88.3 \\
\end{array}$ & 0.063 \\
\hline Fruit juices & $\begin{array}{l}\text { Seldom/never } \\
\text { Once per week } \\
\text { Several time per week } \\
\text { Every day }\end{array}$ & $\begin{array}{l}29.8 \\
17.0 \\
41.8 \\
11.3 \\
\end{array}$ & $\begin{array}{c}33.3 \\
13.6 \\
44.4 \\
8.6\end{array}$ & $\begin{array}{l}25.0 \\
21.7 \\
38.3 \\
15.0\end{array}$ & NS \\
\hline Vegetable or fruit and vegetable juices & $\begin{array}{l}\text { Seldom/never } \\
\text { Once per week } \\
\text { Several time per week } \\
\text { Every day }\end{array}$ & $\begin{array}{c}71.6 \\
7.1 \\
17.7 \\
3.5\end{array}$ & $\begin{array}{c}67.9 \\
8.6 \\
19.8 \\
3.7\end{array}$ & $\begin{array}{c}76.7 \\
5.0 \\
15.0 \\
3.3\end{array}$ & NS \\
\hline $\begin{array}{l}\text { Hot drinks such as tea, coffee, infusions of } \\
\text { herbs or fruit }\end{array}$ & $\begin{array}{l}\text { Seldom/never } \\
\text { Once per week } \\
\text { Several time per week } \\
\text { Every day }\end{array}$ & $\begin{array}{c}4.3 \\
2.8 \\
7.1 \\
85.8 \\
\end{array}$ & $\begin{array}{c}1.2 \\
2.5 \\
3.7 \\
92.6 \\
\end{array}$ & $\begin{array}{c}8.3 \\
3.3 \\
11.7 \\
76.7 \\
\end{array}$ & 0.041 \\
\hline $\begin{array}{l}\text { Sweetened carbonated beverages such as } \\
\text { Coca-Cola, Pepsi, Sprite, Fanta, etc. }\end{array}$ & $\begin{array}{l}\text { Seldom/never } \\
\text { Once per week } \\
\text { Several time per week } \\
\text { Every day }\end{array}$ & $\begin{array}{c}74.5 \\
10.6 \\
12.8 \\
2.1\end{array}$ & $\begin{array}{c}77.8 \\
13.6 \\
8.6 \\
0.0\end{array}$ & $\begin{array}{c}70.0 \\
6.7 \\
18.3 \\
5.0\end{array}$ & 0.038 \\
\hline $\begin{array}{l}\text { Energizing beverages, e.g. 2KC, Black } \\
\text { Horse, Red Bull, Burn, Shot }\end{array}$ & $\begin{array}{l}\text { Seldom/never } \\
\text { Once per week } \\
\text { Several time per week } \\
\text { Every day }\end{array}$ & $\begin{array}{l}90.1 \\
3.5 \\
6.4 \\
0.0 \\
\end{array}$ & $\begin{array}{c}95.1 \\
1.2 \\
3.7 \\
0.0 \\
\end{array}$ & $\begin{array}{c}83.3 \\
6.7 \\
10.0 \\
0.0 \\
\end{array}$ & 0.063 \\
\hline Alcoholic drinks & $\begin{array}{l}\text { Seldom/never } \\
\text { Once per week } \\
\text { Several time per week } \\
\text { Every day }\end{array}$ & $\begin{array}{c}73.8 \\
19.9 \\
6.4 \\
0.0\end{array}$ & $\begin{array}{l}81.5 \\
16.0 \\
2.5 \\
0.0\end{array}$ & $\begin{array}{c}63.3 \\
25.0 \\
11.7 \\
0.0\end{array}$ & 0.024 \\
\hline $\begin{array}{l}\text { Meals eaten outside the home, e.g. in bars, } \\
\text { restaurants, cafes, cafeteria }\end{array}$ & $\begin{array}{l}\text { Seldom/never } \\
\text { Once per week } \\
\text { Several time per week } \\
\text { Every day }\end{array}$ & $\begin{array}{c}53.2 \\
24.1 \\
17.0 \\
5.7\end{array}$ & $\begin{array}{c}61.7 \\
23.5 \\
13.6 \\
1.2 \\
\end{array}$ & $\begin{array}{l}41.7 \\
25.0 \\
21.7 \\
11.7\end{array}$ & 0.014 \\
\hline
\end{tabular}




\section{DISCUSSION}

Our study concerned a specific sub population of patients with T1DM. It was based on the homogeneous group of individuals with all patients being treated with personal insulin pumps. They were characterized with normal BMI, relatively good metabolic control as measured with $\mathrm{HbAlc}$ and good lipid profile. All the patients were also free from advanced late complications of diabetes. However, the dietary habits in studied individuals were far from being optimal. The average score noted in the whole study group as regards the index of healthy diet was $24.2 \pm 10.3$ and our result was not much worse than the Dtuziniak-Golaska et al. 2019 study result $(27.6 \pm 11.1)$. In terms of the index of unhealthy diet in our study, adults achieved better scores in comparison to the children and youth in Dluziniak-Golaska et al. study (15.6 \pm 7.6 vs $22.4 \pm$ 11.1)[5]. In general the scores of dietary habits of the study group with type 1 diabetes are unsatisfactory.

The study showed a difference in dietary index between men and women. Women had higher score in the index of a healthy diet and lower in the unhealthy diet indicator than men. This means that male patients with T1DM may require more educational efforts to make their diet more healthy.

$3 / 4$ of patients declared eating between the main meals. As it turns out, snacks are typical behavior associated with poor nutritional status because they provide excess energy in the form of carbohydrates, [14]. Eating snacks with high fat, sugar and salt may promote the deposition of fatty tissue in the body and worsen the metabolic control $[4,12]$.

Nutritional characteristics of studied group showed that T1DM patients consumed more often white bread and rolls than wholemeal bread and rolls. Similar results were observed in the Gacek et al. from 2017 [6], but the reverse result was observed in the study by Szewczyk et al. from 2011 - this difference may result from a wider age range of the population studied and also may result from the method of conducting the study (only 65\% were T1DM patients, others were T2DM individuals) [13]. The daily consumption of wholemeal bread was declared by $21.3 \%$ of patients, white bread was as high as $34.8 \%$. It was shown that men more often than women choose white bread $(\mathrm{p}=0.024)$.

$57.4 \%$ of respondents consumed vegetables every day in their diet. This is higher percentage as compared to Lodefalk et al. from 2006 who reported that $47 \%$ of teenagers with T1DM declared daily consumption of vegetables [10]. Compared to another Polish study, Gacek et al. from 2017 our group less often chosed vegetables in the daily diet [6] - although our population was characterized by the same age and lived in the same region, the difference may be due to the duration of diabetes (in our study $2 / 3$ of the population suffered from diabetes for more than 10 years, where in the study Gacek et al., only 1/3 patients were characterized with such long duration of the disease) [6].

In our study women more often than men choose fiber-rich products. Similar results were obtained by Gacek et al [6]. Over $60 \%$ of women and $50 \%$ of men in our study consumed a fish at least once a week, which is slightly less than in the study by Gacek et al.(over $60 \%$ men and women) [6].

Our patients more often choose white meat (chicken, turkey, rabbit, etc.) than red meat regardless of gender. A similar result was obtained by Gacek et al study [6]. Over $50 \%$ of people consumed dairy products in the diet at least several times a week. In the study by Lodefalk et al, $85 \%$ of adolescents declared eating dairy products every day [7].

Legumes were not too often chosen as a protein source $-61 \%$ of respondents did not eat or ate very rarely.

Men more often than women choose products that were components of the unhealthy diet index such as: sweets $(p=0.024)$, fast food $(p=0.013)$, carbonated beverages $(p=0.038)$, alcohol $(p=0.024)$. In addition, men more often eat meals outside home $(p=0.014)$. It seems that for the male population of patients with T1DM dietary education should be more focused on avoidance/limitation of sweet and salty snacks as well as sweet drinks and alcohol.

Of note in our study none of the indicators of the quality of the diet was significantly related to the glycemic and metabolic control of the examined population. This may result from relatively high declared physical activity in studied population (data not shown) requiring more carbohydrates, including sweets. It may also result from good skills in hyperglycemia avoidance or hyperglycemia prevention with proper insulin dosing.

To summarize, the obtained results show the food choices by adult patients with T1DM treated with a personal insulin pump. The results of this observational study suggest that despite good glycemic and metabolic control a wider education in such population is necessary regarding the selection of health-promoting product groups.

\section{Acknowledgments}

Thanks are expressed to the participants for their contributions to the study.

\section{Conflicts of interest}

The authors declare no conflict of interest. 


\section{REFERENCES}

1. 2019 Guidelines on the management of diabetic patients. A position of Diabetes Poland. Clin Diabetol 2019;8(1):1-95.

2. American Diabetes Association. Standarts of Medical Care in Diabetes - 2019. Diabetes Care 2019;42(1):1204.

3. Antwi-Baffour S., Kyeremeh R., Boateng SO., Annison L., Seidu MA.: Haematological parameters and lipid profile abnormalities among patients with Type-2 diabetes mellitus in Ghana. Lipids Health Dis 2018 Dec 13;17(1):283.

4. Barnes TL., French SA., Harnack LJ., Mitchell NR., Wolfson J.: Snacking behaviors, diet quality, and body mass index in a community sample of working adults. J Acad Nutr Diet 2015;115(7):1117-1123.

5. Dłużniak-Golaska K., Panczyk M., Szostak-Węgierek D., Szypowska A., Sińska B.Analysis of the diet quality and dietary habits of children and adolescents with type 1 diabetes. Diabetes Metab Syndr Obes. 2019;12:161170.

6. Gacek M., Fraczek B.: Wybory żywieniowe i inne aspekty stylu życia grupy osób z cukrzycą typu 1.[ Dietary choices and other aspects of lifestyle in a group of individuals with type 1 diabetes mellitus]. Probl Hig Epidemiol 2017, 98(1): 33-39 (in Polish).

7. Homma TK., Endo CM., Saruhashi T., Mori AP., Noronha RM., Monte O., Calliari LE.: Dyslipidemia in young patients with type 1 diabetes mellitus. Arch Endocrinol Metab 2015;59(3):215-219.

8. Jeżewska-Zychowicz M., Gawęcki J., Wądołowska L., Czarnocińska J., Galiński G, Kołłajtis-Dołowy A., Roszkowski W., Wawrzyniak A., Przybyłowicz K., Krusińska B., Hawrysz I., Stowińska MA., Niedźwiedzka $E$.: Kwestionariusz do badania poglądów i zwyczajów żywieniowych dla osób w wieku od 16 do 65 lat, wersja 1.2 - kwestionariusz do samodzielnego wypełnienia przez Respondenta. Rozdz. 2. [w:] Kwestionariusz do badania poglądów i zwyczajów żywieniowych oraz procedura opracowania danych. Gawęcki J (red). PAN, Warszawa 2014:1-51 (in Polish).

9. Lin HP., Baghdasarian S., Singer MR., Mott MM., Bradlee ML., Pickering RT., Moore LL.:Dietary Cholesterol, Lipid Levels, and Cardiovascular Risk among Adults with Diabetes or Impaired Fasting Glucose in the Framingham Offspring Study. Nutrients 2018 Jun 14;10(6). pii: E770. doi: 10.3390/nu10060770.

10. Lodefalk M., Aman J.. Food habits, energy and nutrient intake in adolescents with Type 1 diabetes mellitus. Diabet Med 2006;23(11):1225-1232.

11. Ng DS.: Diabetic dyslipidemia: From evolving pathophysiological insight to emerging therapeutic targets. Can J Diabetes 2013;37(5):319-326.

12. O'Connor L., Brage S., Griffin SJ., Wareham NJ., Forouhi NG.: The cross-sectional association between snacking behaviour and measures of adiposity: the Fenland Study, UK. Br J Nut 2015;114:1286-1293.

13. Szewczyk A., Białek A., Kukielczak A., Czech N., Kokot T., Muc-Wierzgoń M., Nowakowska-Zajdel E., Klakla $K$ : Ocena sposobu żywienia osób chorujących na cukrzycę typu 1 i 2 . [Evaluation of eating habits in a group of people with type 1 and 2 diabetes]. Probl Hig Epidemiol 2011;92(2):267-271 (in Polish).

14. Yan MR., Parsons A., Whalley GA., Rush EC.: Effects of a healthier snack on snacking habits and glycated $\mathrm{Hb}$ (HbA1c): a 6-week intervention study. Br J Nutr 2016;116(12):2169-2174.

15. Young KA., Maturu A., Lorenzo C., Langefeld CD., Wagenknecht LE., Chen YI., Taylor KD., Rotter JI., Norris JM., Rasouli N.:The triglyceride to high-density lipoprotein cholesterol (TG/HDL-C) ratio as a predictor of insulin resistance, $\beta$-cell function, and diabetes in Hispanics and African Americans. J Diabetes Complications 2019;33(2):118-122.

Received: 16.04 .2019

Accepted: 30.07.2019

This article is available in Open Access model and licensed under a Creative Commons Attribution-Non Commercial 3.0.Poland License (CC-BY-NC) available at: http://creativecommons.org/licenses/by-nc/3.0/pl/deed.en 